\title{
Screening and brief intervention for drug use in primary care: the Assessing Screening Plus brief Intervention's Resulting Efficacy to stop drug use (ASPIRE) randomized trial
}

\author{
Richard Saitz ${ }^{1,2^{*}}$, Tibor P Palfai ${ }^{3}$, Debbie M Cheng ${ }^{1,4}$, Daniel P Alford ${ }^{1}$, Judith A Bernstein ${ }^{5}$, \\ Christine A Lloyd-Travaglini ${ }^{4,6}$, Seville M Meli ${ }^{1}$, Christine E Chaisson ${ }^{4,6}$, Jeffrey H Samet ${ }^{1,5}$
}

From International Network on Brief Interventions for Alcohol and Other Drugs (INEBRIA) Meeting 2013 Rome, Italy. 18-20 September 2013

\section{Background}

The efficacy of universal screening and brief intervention (SBI) for drug use among primary care (PC) patients is unknown.

\section{Methods}

In this randomized trial (the Assessing Screening Plus brief Intervention's Resulting Efficacy to stop drug use (ASPIRE) study) we tested the efficacy of a brief negotiated interview (BNI), and an adaptation of motivational interviewing (AMI), compared to no BI. Primary care patient participants had Alcohol, Smoking and Substance Involvement Screening Test (ASSIST) drug specific scores of $\geq 4$. Primary outcome at 6 months was number of days use of the drug of most concern (DOMC) in the past 30 days.

\section{Results}

Of 528 subjects, DOMC was: marijuana 63\%, opioid 17\% (prescription opioid 11\%), and cocaine 19\%. ASSIST score was $\geq 27$ (consistent with dependence) for $18 \%$, mean days DOMC use (of 30 days) was 14.4. At 6 months, $98 \%$ completed follow-up and mean days DOMC use was 14.0. Mean adjusted days use of the DOMC at 6 months (negative binomial regression) was 11.5 (no BI) vs. 11.2 (BNI) (incidence rate ratio (IRR) $0.97,95 \% \mathrm{CI} 0.77-1.22$ ) and 12.1 (AMI) (IRR 1.05, 95\% CI 0.84-1.32) ( $\mathrm{p}=0.81$ for both

\footnotetext{
* Correspondence: rsaitz@bu.edu

${ }^{1}$ Clinical Addiction Research and Education (CARE) Unit, Section of General Internal Medicine, Boston University School of Medicine and Boston Medical Center, Boston, Massachusetts, USA

Full list of author information is available at the end of the article
}

comparisons vs. no BI). There were also no significant effects in analyses stratified by DOMC or ASSIST score.

\section{Conclusions}

In this trial, BNI and AMI did not have efficacy for decreasing drug use. If other trials yield consistent results, widespread implementation of drug screening and BI should be reconsidered, and research should focus on alternative ways to address drug use and consequences in primary care settings. Funding was provided by the US National Institutes of Health (National Institute on Drug Abuse and National Center for Advancing Translational Sciences).

\section{Authors' details \\ ${ }^{1}$ Clinical Addiction Research and Education (CARE) Unit, Section of General Internal Medicine, Boston University School of Medicine and Boston Medical Center, Boston, Massachusetts, USA. ${ }^{2}$ Department of Epidemiology, Boston University School of Public Health, Boston, Massachusetts, USA. ${ }^{3}$ Department of Psychology, Boston University College of Arts and Sciences, Boston, Massachusetts, USA. ${ }^{4}$ Department of Biostatistics, Boston University School of Public Health, Boston, Massachusetts, USA. ${ }^{5}$ Department of Community Health Sciences, Boston University School of Public Health, Boston, Massachusetts, USA. ${ }^{6}$ Data Coordinating Center, Boston University School of Public Health, Boston, Massachusetts, USA.}

Published: 4 September 2013

doi:10.1186/1940-0640-8-S1-A61

Cite this article as: Saitz et al:: Screening and brief intervention for drug use in primary care: the Assessing Screening Plus brief Intervention's Resulting Efficacy to stop drug use (ASPIRE) randomized trial. Addiction Science \& Clinical Practice 2013 8(Suppl 1):A61.

\section{() Biomed Central}

@ 2013 Saitz et al; licensee BioMed Central Ltd. This is an Open Access article distributed under the terms of the Creative Commons Attribution License (http://creativecommons.org/licenses/by/2.0), which permits unrestricted use, distribution, and reproduction in any medium, provided the original work is properly cited. 